\title{
Evaluación de la escala de BISAP en el pronóstico de la pancreatitis aguda*
}

\author{
Drs. ADRIÁN MURILlO Z. ${ }^{1}$, PABLO D. MURAKAMI M. ${ }^{1}$, SERGIO A. TOLEDO V. ${ }^{1}$, \\ CARLOS M. CÁRDENAS S. ${ }^{1}$, ENRIQUE R. JEAN S. ${ }^{1}$ \\ 1 Departamento de Cirugía General, Centro Médico ABC I.A.P. \\ México D.F, México.
}

\begin{abstract}
Assessment of a predictive score for complications and mortality among patients with acute pancreatitis
\end{abstract}

Background: It is important to stratify patients with acute pancreatitis according to their risk. Many staging systems for acute pancreatitis have been proposed, each with advantages and disadvantages. Aim: To evaluate the sensitivity, specificity, positive and negative predictive value of the BISAP system. Material and Methods: Retrospective crosses sectional study, which included all patients that presented with acute pancreatitis to a private hospital in Mexico City from 2003 to 2009. The variables needed to calculate BISAP and complications were obtained from the medical records. Results: The records of 345 patients with a mean aged of 52 years $(57 \%$ males) were analyzed. Twelve percent had complications and $2.3 \%$ died. Patients with a BISAP score over three had a significantly higher mortality. The sensitivity, specificity and positive predictive value of a BISAP score over three for mortality were 12.5, 92.9 and 4\%, respectively. Conclusions: Because of its high negative predictive value and specificity the BISAP system can be used to stratify patients who are at low risk for complications related to pancreatitis. If the patient has a score of 3 or higher it is necesary to use other scoring systems which are more sensitive and have a higher positive predictive value.

Key words: BISAP, acute pancreatitis, mortality.

\section{Resumen}

La pancreatitis aguda afecta aproximadamente a 200.000 personas por año en los Estados Unidos. Puede cursar de manera leve o de manera agresiva con una morbi-mortalidad alta. Es importante estratificar a los pacientes de manera temprana de acuerdo a su riesgo. Nosotros analizamos el sistema BISAP y determinamos su sensibilidad, especificidad, valor predictivo positivo y negativo en relación a morbi-mortalidad. Métodos: Realizamos un estudio transversal retrospectivo e incluimos a todos los pacientes con diagnóstico de pancreatitis aguda en un hospital privado de tercer nivel en la ciudad de México desde el 2003 a 2009 . Se obtuvo la morbi-mortalidad y las variables necesarias para calcular el BISAP. Se estratificaron los pacientes. Se utilizó la prueba de Fisher para comparación de variables. Resultados: 345 pacientes fueron incluidos.

*Recibido el 3 de Enero de 2010 y aceptado para publicación el 29 de Abril de 2010.

No hubo fuente de apoyo financiero.

No hay conflicto de interés.

Correspondencia: Dr. Adrián Murillo Z.

Huichapan número 20 interior 2. Colonia Condesa, México D.F., México. Código Postal 06100.

E-mail: dradrianmurillo@gmail.com 
Edad promedio de 51,8 años. La morbilidad fue de 11,6\% y la mortalidad 2,3\%. Conforme aumentaba el puntaje BISAP había una tendencia hacia más morbilidad. Dividimos la población en un grupo de BISAP bajo $(<3)$ y un grupo con BISAP alto $(>=3)$ y observamos una diferencia estadísticamente significativa en cuanto a la morbilidad de ambos grupos. El BISAP tiene una alta especificidad y valor predictivo negativo. Su sensibilidad y valor predictivo positivo son pobres. Conclusiones: Dado su alto valor predictivo negativo y especificidad el BISAP se puede utilizar para estratificar los pacientes que tienen un bajo riesgo de presentar pancreatitis aguda severa relacionada con morbilidad. Si el paciente examinado presenta un BISAP $>=$ 3, creemos necesario utilizar otro sistema con más sensibilidad y valor predictivo positivo para el manejo.

Palabras clave: Pancreatitis, factores de pronóstico, BISAP.

\section{Introducción}

La pancreatitis aguda afecta aproximadamente a 200.000 pacientes cada año en los Estados Unidos ${ }^{1,2}$. Aproximadamente $85 \%$ de los pacientes afectados cursan con una pancreatitis intersticial, el 15\% restante cursa con una pancreatitis necrotizante. El $10 \%$ de los pacientes con pancreatitis y el 29 $78 \%$ de los pacientes con pancreatitis necrotizante presentarán falla orgánica. La mortalidad va desde un $3 \%$ en la pancreatitis intersticial hasta $17 \%$ en la necrotizante. La mortalidad también varía según la presencia de falla orgánica, siendo de $0 \%$ en ausencia de falla orgánica, $3 \%$ con falla de un solo órgano y $47 \%$ con la falla multiorgánica ${ }^{3}$.

Es importante detectar y estadificar a los pacientes con pancreatitis aguda para poder decidir quiénes requieren de una unidad de cuidados intensivos. Se han descrito diversos sistemas para estadificar la severidad de la pancreatitis y así mejorar el pronóstico, entre estos la clasificación de Ranson, APACHE II, el índice de severidad por tomografía computada (CTSI), y el BISAP.

La clasificación de Ranson tiene poco poder predictivo para determinar la severidad de la pancreatitis ${ }^{4}$. Además tiene la desventaja que requiere de $48 \mathrm{~h}$ para completarse, por lo que se puede perder tiempo valioso en estadificar el riesgo del paciente.

La clasificación de APACHE II es la que actualmente acepta la Sociedad de Gastroenterología Americana para la estratificación de pancreatitis aguda $^{3}$. El puntaje incluye variables de laboratorio y fisiológicas. Se acepta como una pancreatitis grave aquella que cursa con un puntaje total $>8$. Tiene la ventaja sobre la escala de Ranson que no requiere de $48 \mathrm{~h}$ para generarse, y se puede determinar de manera seriada ayudando a dar seguimiento a la evolución del padecimiento. Aun así, este sistema no es específico para pancreatitis aguda, y contiene algunos parámetros que no son relevantes en determinar el pronóstico de la pancreatitis.

El CTSI es un índice que se determina según el grado de inflamación pancreática, el número de colecciones y la presencia o ausencia de necrosis, determinada por el realce con contraste intravenoso
(IV). Es útil en valorar los efectos locales de la pancreatitis, sin embargo, no refleja de manera adecuada la respuesta sistémica inflamatoria ${ }^{5}$. Además, el uso rutinario de la tomografía al momento del ingreso de un paciente con pancreatitis aguda no está avalado por la Sociedad de Gastroenterología Americana. Sólo la recomiendan en caso de duda diagnóstica al momento de la valoración inicial, o unos días después de la admisión para distinguir una pancreatitis intersticial de una necrotizante. Otra desventaja que tiene es que para completar la valoración del CTSI se requiere el uso de medio de contraste IV, lo que requiere de un valor de creatinina sérico menor a $1,5 \mathrm{mg} / \mathrm{dl}$.

Recientemente se propuso como alternativa el BISAP, un sistema que evalúa 5 puntos: 1) Nitrógeno de Urea $>25 \mathrm{mg} / \mathrm{dl}$; 2) alteración del estado mental evidenciado por desorientación; 3) presencia de respuesta inflamatoria sistémica (2 o más de las siguientes variables: frecuencia cardíaca $>90$ latidos/min, frecuencia respiratoria $>20$ por min, o $\mathrm{PaCo} 2<32 \mathrm{mmHg}$, temperatura $>38$ o $<36^{\circ} \mathrm{C}$, y leucocitos $>12.000 \mathrm{o}<4.000$ células por $\mathrm{mm}^{3} \mathrm{o}$ $>10 \%$ bandas); 4) derrame pleural en radiografía de tórax o estudio tomográfico, y 5) edad $>60$ años ${ }^{6}$.

$\mathrm{Wu}$ et al, observaron que pacientes con 2 o menos puntos tenían una mortalidad $<1 \%$. Aquellos con BISAP $>=3$ tuvieron mortalidad entre 5-20\%. En el mismo estudio, los autores concluyen que el sistema puede predecir de manera temprana la mortalidad en aquellos pacientes que no tienen falla orgánica temprana.

En el estudio de Wu et al, se comparó el BISAP con el APACHE II y no se observó diferencia estadísticamente significativa entre ambos en su poder predictivo. Papachristou et al, compararon el BISAP, APACHE II, Ranson y CTSI. Determinaron la sensibilidad, especificidad, valor predictivo positivo (VPP) y valor predictivo negativo (VPN) de cada uno de los sistemas predictivos en relación a la capacidad para discernir severidad, necrosis pancreática y mortalidad. El BISAP tuvo una sensibilidad para predecir severidad, necrosis pancreática y mortalidad ligeramente por debajo de los tres sistemas convencionales. Sin embargo, en 
cuanto a su especificidad en estos tres rubros fue ligeramente mayor.

El objetivo principal de este estudio fue determinar la sensibilidad, especificidad, VPP y VPN del BISAP en relación a la mortalidad y morbilidad de los pacientes con pancreatitis aguda.

\section{Material y Método}

Se realizó un estudio retrospectivo, transversal comparativo de los pacientes con pancreatitis aguda que se presentaron a un hospital de tercer nivel privado en la ciudad de México desde enero de 2003 a diciembre de 2009. Se realizó una búsqueda en el sistema de expedientes electrónico del hospital utilizando como criterio de búsqueda el diagnóstico de pancreatitis aguda. Se obtuvo de cada uno de los expedientes las variables demográficas de la población y las correspondientes al sistema BISAP. Se clasificaron los pacientes de acuerdo a su puntuación (0-5). Se obtuvieron las variables de mortalidad y morbilidad. Se definió falla orgánica según los criterios de Atlanta de 1992. Se excluyó todo paciente que no cumpliera con el diagnóstico de pancreatitis aguda o aquellos en los que no se obtuvieron al ingreso las variables necesarias para calcular el BISAP. Se utilizó la prueba de Fisher para comparar variables dicotómicas.

\section{Definiciones}

Pancreatitis aguda: 2 o más de los siguientes: dolor abdominal característico; elevación de amilasa y/o lipasa 3 veces por arriba del límite de lo normal; y/o estudio tomográfico con cambios consistentes de pancreatitis aguda.

Alteración en el estado neurológico: Cualquier deterioro neurológico agudo comparado con el estado neurológico basal del paciente relacionado al inicio de la sintomatología abdominal.
Presencia/ausencia de derrame pleural: Determinado por hallazgos tomográficos, radiológicos, o en ausencia de ambos, hallazgos clínicos consistentes con derrame pleural (matidez a la percusión, disminución del murmullo vesicular a la auscultación).

Morbilidad: Las complicaciones locales y/o sistémicas que se originaron como consecuencia de la pancreatitis aguda, que no estaban presentes o no se detectaron al momento del ingreso a hospitalización.

\section{Análisis estadístico}

Se utilizó estadística descriptiva en la presentación de los datos. Para el análisis se utilizó la prueba de Fisher, y se tomó como resultado estadísticamente significativo una $p<0,05$. Se calculó sensibilidad, especificidad, VPP y VPN.

\section{Resultados}

Se obtuvieron 382 pacientes de la base de datos con diagnóstico de pancreatitis aguda. De estos se excluyeron 37 pacientes que no cumplieron con los criterios de inclusión, quedando 345 pacientes para el estudio. La edad promedio fue de 51,8 años. Ciento noventa y cinco $(56,6 \%)$ fueron de sexo masculino. La morbilidad fue de $11,6 \%(n=40)$ y la mortalidad general fue de $2,3 \%(n=8)$. La estancia hospitalaria promedio fue de 7,4 días. La morbilidad relacionada a pancreatitis aguda se presentó en 40 pacientes. Se detalla en la Tabla 1, según el grado de BISAP.

En la Tabla 2 se resume la morbilidad y mortalidad según el BISAP. Los resultados de la sensibilidad, especificidad, VPP y VPN en relación a morbilidad y mortalidad del BISAP se calcularon tomando como valor de corte un puntaje de $3 \mathrm{y}$ se muestran en las Tablas 3 y 4.

Tabla 1. Puntaje de BISAP y tipo de morbilidad asociada

\begin{tabular}{|c|c|c|c|c|c|}
\hline Morbilidad & $\begin{array}{c}\text { BISAP 0 } \\
\% \quad(n)\end{array}$ & $\begin{array}{c}\text { BISAP 1 } \\
\% \quad(n)\end{array}$ & $\begin{array}{c}\text { BISAP 2 } \\
\% \quad(n)\end{array}$ & $\begin{array}{c}\text { BISAP 3 } \\
\% \quad(n)\end{array}$ & $\begin{array}{c}\text { BISAP } 4 \\
\% \quad(n)\end{array}$ \\
\hline Complicaciones locales & $5,8 \quad(8)$ & $1,47(2)$ & $6,38(3)$ & $5,00 \quad(1)$ & 0 \\
\hline Requirieron drenaje de absceso y/o debridación & 2,19 (3) & $0,74(1)$ & $6,38(3)$ & $5,00 \quad(1)$ & 0 \\
\hline Falla orgánica única & $0,73(1)$ & $2,22(3)$ & $8,51(4)$ & $5,00 \quad(1)$ & 0 \\
\hline Falla orgánica múltiple & $0,73(1)$ & $3,68(5)$ & $4,26(2)$ & $15,00(3)$ & $60,00(3)$ \\
\hline Otras & 0 & $0,74(1)$ & $4,26(2)$ & 0 & 0 \\
\hline
\end{tabular}


Tabla 2. Puntaje de BISAP, número de pacientes, morbilidad y mortalidad relacionada

\begin{tabular}{crll}
\hline BISAP & \multicolumn{1}{c}{$\mathbf{n}$} & Mortalidad & Morbilidad \\
\hline 0 & 137 & $1(0,7 \%)$ & $10(7,2 \%)$ \\
1 & 136 & $3(2,2 \%)$ & $11(8,1 \%)$ \\
2 & 47 & $3(6,3 \%)$ & $12(25,5 \%)$ \\
3 & 20 & $1(5 \%)$ & $4(20,0 \%)$ \\
4 & 5 & 0 & $3(60 \%)$ \\
5 & 0 & 0 & 0 \\
\hline
\end{tabular}

Tabla 3. Sensibilidad, especificidad, VPP, VPN de BISAP menor $o$ igual a 2, o mayor de 3 en relación a morbilidad

\begin{tabular}{cccc}
\hline Sensibilidad & Especificidad & VPP & VPN \\
\hline $17,5 \%$ & $94 \%$ & $28 \%$ & $89,6 \%$ \\
\hline
\end{tabular}

Tabla 4. Sensibilidad, especificidad, VPP, VPN de BISAP menor $o$ igual a 2, o mayor de 3 en relación a mortalidad

\begin{tabular}{cccc}
\hline Sensibilidad & Especificidad & VPP & VPN \\
\hline $12,5 \%$ & $92,9 \%$ & $4 \%$ & $97,8 \%$ \\
\hline
\end{tabular}

\section{Discusión}

Nuestro estudio mostró que las características de la población con pancreatitis aguda que fue hospitalizada durante el período establecido, es similar a la reportada por otros autores tanto en relación a proporción masculino femenino como a la mortalidad asociada a la enfermedad ${ }^{5}$.

El BISAP se ha comparado ya con las escalas pronósticas más comúnmente utilizadas, y se ha visto que éste refleja alteraciones sistémicas en el individuo, y tiene poca capacidad de predecir complicaciones locales ocasionadas por la pancreatitis. Se ha tomado como valor de cohorte para determinar un BISAP bajo (puntaje de 0,1 y 2) y un puntaje alto (mayor o igual a 3$)^{5,7}$. La aplicación del BISAP en la población estudiada mostró que la mayor parte de los pacientes hospitalizados cursaron con una pancreatitis con poca alteración sistémica, reflejada por una puntuación de BISAP baja. Este mismo segmento de la población presentó una morbilidad menor que aquellos pacientes en los que se presentó un BISAP mayor.

Hubo una tendencia conforme aumentaba el puntaje del BISAP a haber mayor morbilidad. Hubo una diferencia significativa en la morbilidad entre los pacientes que presentaron un puntaje de 1 en relación a $2(p=0,0039)$, no hubo diferencia estadísticamente significativa entre el grupo 2 en relación a $3(p=0,759)$ y hubo una tendencia de menor morbilidad en aquellos con puntaje de 3 en relación a 4 , sin llegar a ser significativa estadísticamente $(\mathrm{p}=$ $0,1130)$.

De forma interesante, en la Tabla 1 observamos que puntajes de BISAP bajos se ven asociados más frecuentemente a complicaciones locales que no requieren intervención (drenaje). Conforme el puntaje de BISAP aumenta, se observa que aumenta el porcentaje de pacientes que presentan falla orgánica múltiple. Además, las complicaciones locales que se generaron, requirieron intervención quirúrgica.

$\mathrm{Al}$ dividir los resultados en pacientes con un BISAP bajo en relación a alto y comparar la morbilidad, encontramos una diferencia estadísticamente significativa entre ambos grupos ( $p<0,0166$, Odds Ratio 3,37 (95\% Intervalo de Confianza 1,31-8,67)). Esto es coherente con una de las críticas de Papachristou et al a los sistemas predictivos, en que son útiles en los extremos, más, pierden su valor predictivo en grados intermedios de la pancreatitis ${ }^{5}$. Singh, reporta en su estudio, que los pacientes con BISAP mayor o igual a tres tienen 7,4 veces más probabilidad de tener falla orgánica y 3,8 veces más posibilidad de tener necrosis pancreática. Es evidente que un BISAP elevado cursa con mayor posibilidad de presentar este tipo de complicaciones ${ }^{7}$.

La mortalidad baja $(\mathrm{n}=8)$ que se observó, limita las conclusiones que se pueden derivar de cada puntaje de BISAP. Al dividir los pacientes en BISAP bajo y alto, no obtuvimos una diferencia estadísticamente significativa entre ambos grupos $(p=0,46)$.

Observamos que el sistema de BISAP cuenta con alta especificidad y un alto VPN en relación a la presentación de morbilidad o mortalidad. Sin embargo, también observamos que es un estudio con una sensibilidad y VPP pobre. Esto se traduce en que un resultado de BISAP alto es pobre en predecir la ocurrencia de morbilidad o mortalidad. Sin embargo, un puntaje bajo es un buen indicador para predecir aquellos pacientes con pancreatitis que no van a cursar con complicaciones o mortalidad.

Es posible que la baja especificidad y bajo VPP sea en parte un reflejo de aquellos pacientes que en algún momento de su hospitalización cursaron con una falla orgánica única y transitoria (lo que no necesariamente se traduce a una condición que amenaza la vida del paciente) $)^{8}$, los cuales en nuestro estudio 
clasificamos como morbilidad, y que no necesariamente equivalen a una pancreatitis severa. En el estudio de Papachristou et al, también se observó que la sensibilidad y el VPP del BISAP eran bajos $(37,4$ y 57,7 respectivamente) en cuanto a la predicción de severidad. De la misma manera obtuvieron una baja sensibilidad y VPP en relación a la mortalidad (57,1 y 15,4 respectivamente).

Nuestro estudio tiene ciertas limitaciones. El número de pacientes con un BISAP de 4 y 5 es muy bajo, por lo que la muestra no es del todo representativa, y entre estos no hubo mortalidad. Por otro lado, no se les realizó estudios de imagen a todos los pacientes dentro de las primeras $24 \mathrm{~h}$, por lo que tuvimos que contar con criterios clínicos (menos sensibles) para determinar la presencia de derrame pleural, lo que pudo ocasionar que algunos pacientes fueran estratificados con un BISAP más bajo de lo real.

En conclusión, dada su alta especificidad y valor VPN, pensamos que el BISAP puede ser utilizado como un sistema sencillo, que no aumenta el costo de la hospitalización, para identificar en las primeras $24 \mathrm{~h}$ a los pacientes que tienen un bajo riesgo de cursar con una pancreatitis grave. Sin embargo, pacientes que obtienen un BISAP igual o mayor a tres, o aquellos que empeoran a pesar del manejo médico inicial requieren de la aplicación de otros métodos para estratificar de manera más fidedigna su riesgo (particularmente el APACHE II y el CTSI).

\section{Agradecimientos}

A Dr. Jorge Cervantes Castro.

\section{Nota de Pares Revisores}

El trabajo está bien diseñado. Presenta un sistema de Score para evaluar la gravedad de la respuesta inflamatoria de la Pancreatitis Aguda. Tiene el mérito de mostrar que cuando la enfermedad es grave, el Score pierde sensibilidad y valor predictivo. BISAP no parece tener mayor utilidad, al compararlo con el difundido APACHE II y el emergente SOFA.

\section{Referencias}

1. Swaroop VS, Chari ST, Clain JE. Severe acute pancreatitis. JAMA 2004; 291: 2865-2868.

2. Papachristou GI, Clermont G, Sharma A, Yadav D, Whitcomb DC. Risk and markers of severe acute pancreatitis. Gastroenterol Clin North Am 2007; 36: 277 296.

3. Banks PA, Freeman ML, Practice Parameters Comite of the American College of Gastroenteroology. Practice Guidelines in Acute Pancreatitis. Am J Gastroenterol 2006; 101: 2379-2400.

4. De Bernardinis M, Violi V, Roncoroni L, Boselli AS, Giunta A, Peracchia A. Discriminating power and information content of Ranson's prognostic signs in acute pancreatitis: A meta-analytic study. Crit Care Med 1999; 27: 2272-2283.

5. Papachristou GI, Mundana V, Yadav D, O'Connell M, Sanders MK, Slivka A, et al. Comparison of BISAP, Ranson's, APACHE II, and CTSI Scores in Predicting Organ Failure, Complications, and Mortality in Acute Pancreatitis. Am J Gastroenterol advance online publication 27 October 2009; doi: 10.1038/ajg.2009.622.

6. Wu BU, Johannes RS, Sun X, Tabak Y, Conwell DL, Banks PA. The early prediction of mortality in acute pancreatitis: a large population-based study. Gut 2008; 57: 1698-1703.

7. Singh VK, Wu BU, Bollen TL, Repas K, Maurer R, Johannes RS, et al. A Prospective Evaluation of the Bedside Index for Severity in Acute Pancreatitis Score in Assessing Mortality and Intermediate Markers of Severity in Acute Pancreatitis. Am J Gastroenterol 2009; 104: 966-971.

8. Rau BM. Outcome determinants in Acute Pancreatitis. The American Journal of Surgery 2007; 194: S39-S44. 